\title{
Robust Mobile Robot Localization based on Security Laser Scanner
}

\author{
Héber Sobreira, A. Paulo Moreira, Paulo Gomes Costa \\ INESC TEC (formerly INESC PORTO) - Robotics and \\ Intelligent Systems, Faculty of Engineering of University of \\ Porto \\ Rua Dr. Roberto Frias, 4200-465 Porto, Portugal \\ Portugal \\ heber.m.sobreira@inescporto.pt, amoreira@fe.up.pt, \\ paco@fe.up.pt
}

\begin{abstract}
This paper addresses the development of a new localization system based on a security laser presented on most AGVs for safety reasons. An enhanced artificial beacons detection algorithm is applied with a combination of a Kalman filter and an outliers rejection method in order to increase the robustness and precision of the system. This new robust approach allows to implement such system in current AGVs. Real results in industrial environment validate the proposed methodology.
\end{abstract}

Keywords-AGV, mobile robotics, localization, artificial beacons, Kalman filter, outliers rejection, security laser

\section{INTRODUCTION}

One of the most important requirement of an industrial mobile robot is a robust self-localization. Basically, it can be defined as the task of estimating the robot's pose in a map of the environment. This task has captured the attention of researches and developers of mobile robots over the last years. There are some solutions adopted that solves the localization problem but bring some disadvantages [1][2].

One of the common approaches is based on floor line followers (colored or magnetic). This solution is limited with the rigid path becoming difficult and expensive to change. Other solution is based on lasers and artificial landmarks placed on specific places. In some circumstances this solution requires a laser scanner and a robot with a minimum height, in order to avoid occlusions of the landmarks. There are also some mechanical problems that invalidate this approach: for example robots that transport structures on the top (as seen in Fig. 1). The robot must localize itself with landmarks near the floor. This robot only possesses a security laser on the lower position. This approach allows developing new small AGV that improves flexibility.

This type of situation requires the improvement of the current systems (as actually exist) with new approaches that could be used in an industrial environment. On the other hand, one of the main security requirements of industrial mobile robots is the use of security lasers, preventing collisions with obstacles and humans.

\author{
José Lima \\ INESC TEC (formerly INESC PORTO) - Robotics and \\ Intelligent Systems, Rua Dr. Roberto Frias, 4200-465 Porto, \\ Portugal \\ Polytechnic Institute of Bragança \\ Campus Sta Apolónia, Apartado 1134, 5301-857 Bragança \\ Portugal \\ jllima@ipb.pt
}

Based on the present disadvantages of current localization systems and having in mind the presence of the security lasers in most mobile robots, unlike the other systems this paper presents a solution that uses the security laser to perform the self-localization task in the environment based on a few landmark's number. By this way, it is also possible to reduce the cost of the final robot. The developed algorithm searches the artificial landmarks and avoids undesired outliers.

In our example it was used an S3000 Expert laser scanner from SICK.

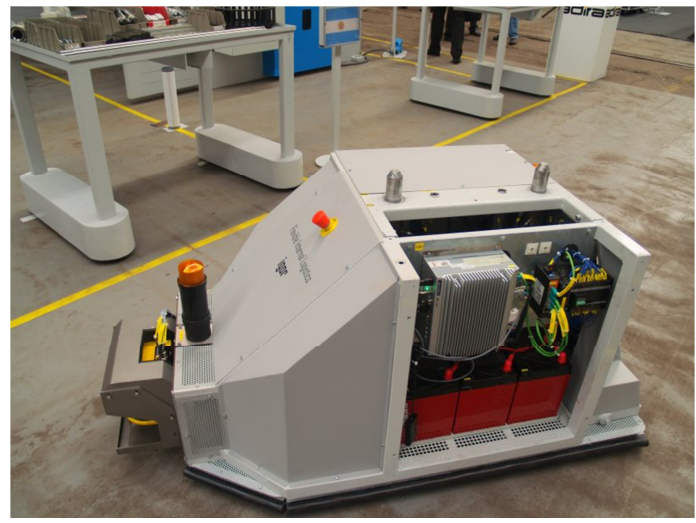

Fig. 1. Robot in the shop floor of an industrial environment.

The system was developed in ROS [3], a well-known Robot Operating System that provides libraries and tools to help software developers create robot applications. The main topic of this work is the robustness of the algorithm to the outliers (other robots, people and objects) that can affect the localization.

Unfortunately, the field of view from the laser is considerably reduced (when compared to a localization laser). The security laser scanner provides 190 degrees whilst a standard localization laser provides 360 degrees of vision. In this work a 180 degrees Field of View (FOV) was used. This drawback can be overcome with accurate and robust algorithms improvement as stated in next sections. 
This paper is organized as follows: after the introduction section, section II addresses the state of the art where related work is described. Section III presents the algorithm whereas section IV addresses the practical results. Finally, section V rounds up with conclusions and points out some future work.

\section{RELATED WORK}

Over the last two decades researches have been working in mobile robot localization [7]. There are a huge variety of solutions based on several approaches. Unfortunately, commercial solutions do not include the complete localization system and many only work in well-known and controlled environments. Using lasers it is possible to apply algorithms that finds the match between the information from the laser and the map [4] [11]. But, the related localization systems should be based on indistinguishable beacons. With this criteria, there is the well-known Thrun et al. of book [5] entitled "EKF Localization with Unknown Correspondences" as an extended Kalman filter approach.

The core of this localization algorithm is the identification of the beacons that are indistinguishable between them to find the correspondence position in the map. Several approaches exist on the literature, such as Thrun [5] that uses the "maximum likelihood data estimation" to compute for each iteration and for each beacon the probability density function. The desired solution is the one that maximizes that function. There are other approaches, like Ronzoni et al. [8] that reaches the global localization based on the distance of the reflectors. By this approach encoders odometry data is not used and global positioning is computed without previous information of robot localization.

\section{ALGORITHM}

\section{A. Problem definition}

The main problem for the beacon based localization task can be defined as the estimation of the AGV pose:

$$
\mathrm{X}_{\mathrm{v}}=\left[\begin{array}{lll}
x_{\mathrm{v}} & \mathrm{y}_{\mathrm{v}} & \theta_{\mathrm{v}}
\end{array}\right]^{\mathrm{T}}
$$

Different coordinates are denoted in Fig. 2 where $W$ is the world coordinates whereas $R$ is the AGV coordinates. From the beacons map $\left(\mathrm{M}_{\mathrm{B}}\right)$ it is possible to gather the information about reflectors positions of cylinder form and indistinguishable between them:

$$
\mathrm{M}_{\mathrm{B}}=\left[\begin{array}{lll}
\mathrm{M}_{\mathrm{B}, 1} & \cdots & \mathrm{M}_{\mathrm{B}, \text { numB }}
\end{array}\right]^{\mathrm{T}}
$$

Where $\mathrm{M}_{\mathrm{B}, \mathrm{i}}=\left[\begin{array}{ll}x_{\mathrm{B}, \mathrm{i}} & \mathrm{y}_{\mathrm{B}, \mathrm{i}}\end{array}\right]^{\mathrm{T}}$ is the position of reflector $i$ in $W$ coordinates.

From $Z_{\mathrm{L}}$ observations (laser measures), where $Z_{L, i}$ corresponds to the polar coordinates ( $r_{L, i}$ distance, $\varphi_{L, i}$ angle) of the detected obstacle in the $R$ coordinates, and $c_{L, i}$ is a Boolean related with the reflectivity of the target (a reflector presents high reflectivity):

$$
\mathrm{Z}_{\mathrm{L}}(\mathrm{k})=\left\{\mathrm{Z}_{\mathrm{L}, \mathrm{i}}(\mathrm{k})=\left[\begin{array}{ll}
\mathrm{r}_{\mathrm{L}, \mathrm{i}}(\mathrm{k}) & \phi_{\mathrm{L}, \mathrm{i}}(\mathrm{k})
\end{array}\right]^{\mathrm{T}}, \mathrm{C}_{\mathrm{L}, \mathrm{i}}(\mathrm{k}): \mathrm{i} \in\left[\begin{array}{ll}
1 & \mathrm{numL}
\end{array}\right]\right\}
$$

Based on the odometry information, it is also possible to estimate the position and orientation of the $R$ coordinates (robot pose):

$$
\mathrm{u}(\mathrm{k})=\left[\begin{array}{lll}
\Delta x_{\text {odos }}(\mathrm{k}) & \Delta y_{\text {odos }}(\mathrm{k}) & \Delta \theta_{\text {odos }}(\mathrm{k})
\end{array}\right]^{\mathrm{T}}
$$

This is an input for the Kalman filter as further presented. The mapping task will not be addressed here.

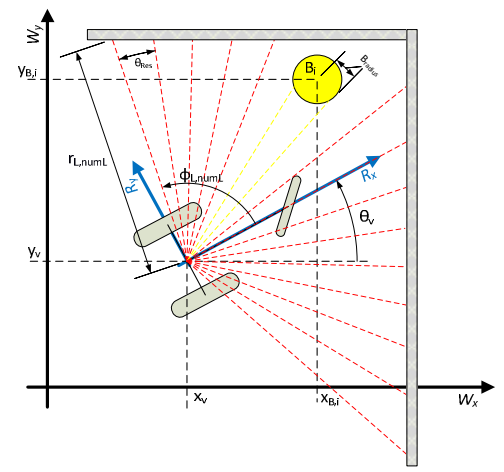

Fig. 2. AGV and world coordinates with beacon map.

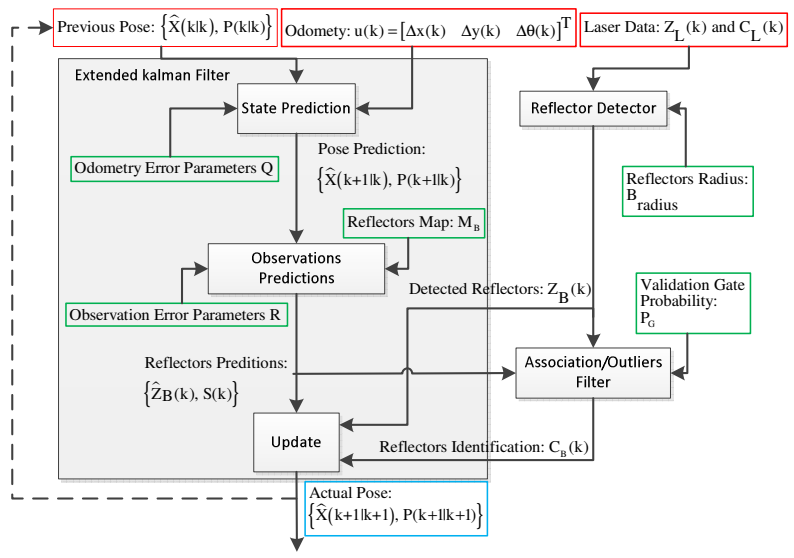

Fig. 3. System architecture.

\section{B. Problem approach}

The problem of localization can be expressed as a block diagram as presented in Fig. 3. Each block presents inputs (red), outputs (blue) and parameters (green). The grey area addresses the sensor fusion algorithm known as the Extended Kalman Filter (EKF).

Next subsection presents the EKF application to perform the localization task based on reflectors and then further subsections address the reflectors detection and filtering.

\section{Extended Kalman Filter}

The EKF is a well-known algorithm applied to sensor fusion in mobile robotics, in this case the distance and angles to the reflectors and odometry. It computes the statistical data related to the state estimation (pose) and also deals with the noise and errors modelling it as a Gaussian signal. Besides, industrial application usually require high precision solutions where Kalman filter fits better in opposition to other approaches such as particle filters [6].

In order to apply an EKF, it is necessary to define the model: state transition $f($.$) and observation h($.$) . As it can be$ expressed through equation $5, f($.$) models the evolution of the$ robot pose based on the last state and odometry $u(k) . Q(k)$ is the noise co-variance and depends on the encoders data. Odometry model was based on [10]. 


$$
\mathrm{X}_{\mathrm{v}}(\mathrm{k}+1)=\left[\begin{array}{c}
x_{\mathrm{v}}(\mathrm{k}+1) \\
\mathrm{y}_{\mathrm{v}}(\mathrm{k}+1) \\
\theta_{\mathrm{v}}(\mathrm{k}+1)
\end{array}\right]=\mathrm{f}\left(\mathrm{X}_{\mathrm{v}}(\mathrm{k}), \mathrm{u}(\mathrm{k})\right)+\mathrm{N}(0, \mathrm{Q}(\mathrm{k}))
$$

Observation $h($.$) models the relative position of reflectors$ as a robot pose function. It is a Gaussian zero average affected noise with a co-variance $R$. In the presented work, $\mathrm{R}$ is constant that depends on the system. For further details see [9].

$$
\left[\begin{array}{c}
r_{B, i} \\
\phi_{B, i}
\end{array}\right]=h\left(M_{B, i}, X_{v}(k)\right)+N(0, R)
$$

Having in mind the transition and observation model it is possible to apply the algorithm as an adaptation of "EKF Localization with Unknown Correspondences" presented in [9].

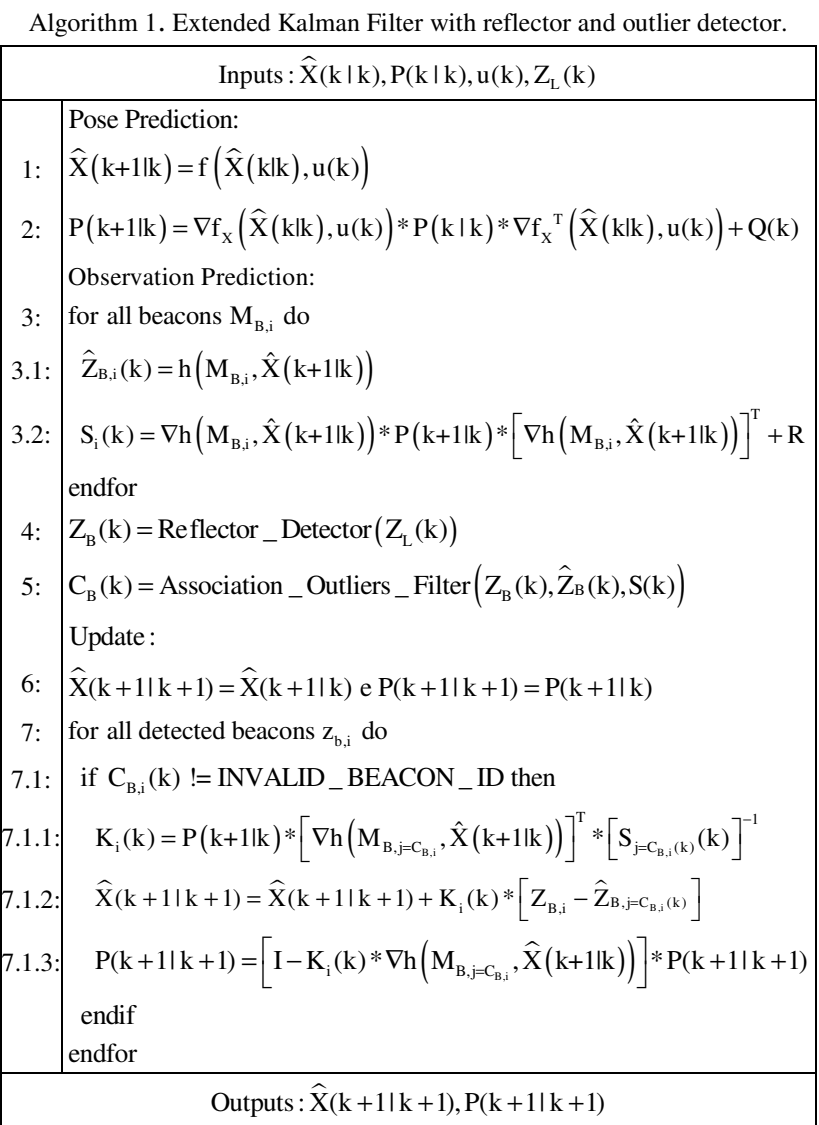

Algorithm 1 describes the processing of the Kalman Filter. As input there is the actual pose $X(k \mid k)$, its co-variance, odometry data $u(k)$ and laser information $Z_{L}(k)$. As output the filter presents the pose estimation with its uncertainty characterized by the co-variance matrix $P(k+1 \mid k+1)$. The algorithm presents the following steps:

1: Prediction of the next pose based on odometry data;

2: Estimation of co-variance matrix of the state error;

3.1 and 3.2:Prediction of observations and co-variance (through observation model);

4: Pre-processing of laser data and reflectors detection/filtering (further presented in subsection D);

5: Reflectors identification: to match detected reflectors and reflectors map (further presented in subsection $\mathrm{E}$ ).
7.1: Rejecting outliers;

7.1.1: Kalman gain. $\mathrm{C}_{\mathrm{B}, \mathrm{i}}(\mathrm{k}) ; \mathrm{C}_{\mathrm{B}, \mathrm{i}}(\mathrm{k})$ is the index's beacons map element $\left(\mathrm{M}_{\mathrm{B}, \mathrm{i}}\right)$ associated to i detection $\left(\mathrm{Z}_{\mathrm{B}, \mathrm{i}}\right)$;

7.1.2: Kalman filter update ;

7.1.3: New co-variance calculation;

\section{Reflector detection/filtering}

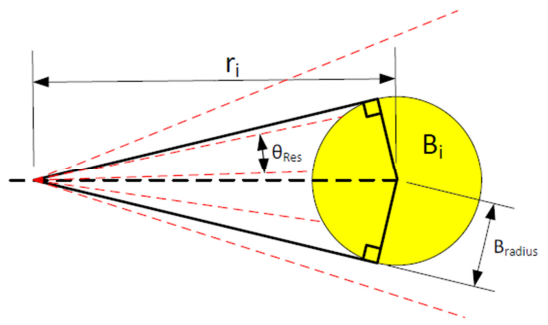

Fig. 4. Laser beams intercepting the beacon.

The reflector detector module processes the acquired data from laser $\mathrm{Z}_{\mathrm{L}}$ detecting reflectors and measures its distances $\mathrm{Z}_{\mathrm{B}}$ related to the robot coordinates. $\mathrm{Z}_{\mathrm{B}}$ provides a set of polar coordinated represented by $\left[r_{i}, \varphi_{i}\right]^{T}$.

Laser provides distance and reflectivity information. Detector/Filter tasks can be defined as:

- Splits $\mathrm{Z}_{\mathrm{L}}$ in clusters depending on reflectivity $C_{L, i}$.

- For each cluster: compute its polar coordinates as a central measure ( $\mathrm{r}$ in equation 6 ).

- Finally, a detector filter is applied to ignore outliers.

The detector filter is based on the geometric relation of (10) that allows to model $M_{\text {num }}$ (the number of the beams). This filter is able to reject other objects than the real beacons reflectors. The first approach is to accept the objects with a constant size (length of the beacon). As the number of beams $\left(\mathrm{M}_{\text {num }}\right)$ depends on the distance of the laser-object a function can be found and is presented in equation (7).

$$
\mathrm{M}_{\text {num }}(\mathrm{r})=\text { floor }\left(\frac{2 * \arcsin \left(\frac{\mathrm{B}_{\text {radius }}}{\mathrm{r}}\right)}{\theta_{\text {Res }}}\right)
$$

Where $B_{\text {radius }}$ is the beacon radius (usually cylinders), $r$ is the distance between laser and the beacon center and $\theta_{\text {Res }}$ is the angular resolution of the laser, as presented in Fig. 4. By this way it is possible to ignore objects with wrong dimensions.

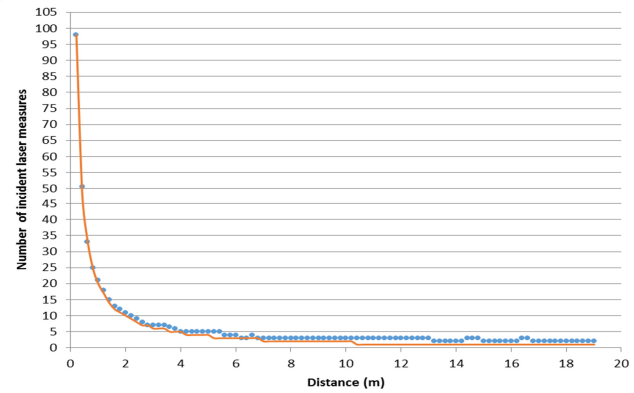

Fig. 5. Comparison between model and measured number of beams for beacons intersection as a distance function (blue circles are measures and red line is the model)

An experimental setup was implemented to validate this approach up to 19 meters of distance between beacons and 
laser. The result is presented in Fig. 5 and it allows to validate the distance-number of beams function that further can be applied.

The error between the number of detected beams and the model allows to reject or accept each cluster. Fig. 6 presents an acquisition data set $\left(Z_{L}\right.$ e $\left.C_{L, i}\right)$ where black points have lower reflection and red points have high reflection. As result, the three reflectors were correctly detected and filtered whereas the two other clusters were rejected.

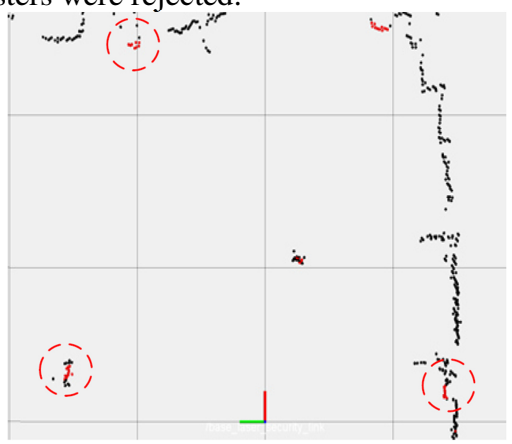

Fig. 6. Laser data: Distance and reflectivity (red points have high reflectivity).

\section{E. Association outliers filter}

The main purpose of this filter is composed by two functions: a) identify the detected reflectors and b) to filter outliers that the previous filter (reflector detector) could miss. This approach is based on the actual estimation of robot pose $X($.$) and P($.$) rejecting measures that depend on its probability.$ This procedure is presented in algorithm 2 , where $Z_{\mathrm{B}}(\mathrm{k})$ are the detected reflectors and $Z_{B}(k)$ and $S(k)$ are the predicted observations and its covariance. As result of this algorithm $C_{B}$ consists in an array where $C_{B, i}$ shows the reflectors map index of $\mathrm{M}_{\mathrm{B}, \mathrm{i}}$ associated to detection $\mathrm{Z}_{\mathrm{B}, \mathrm{i}}$.

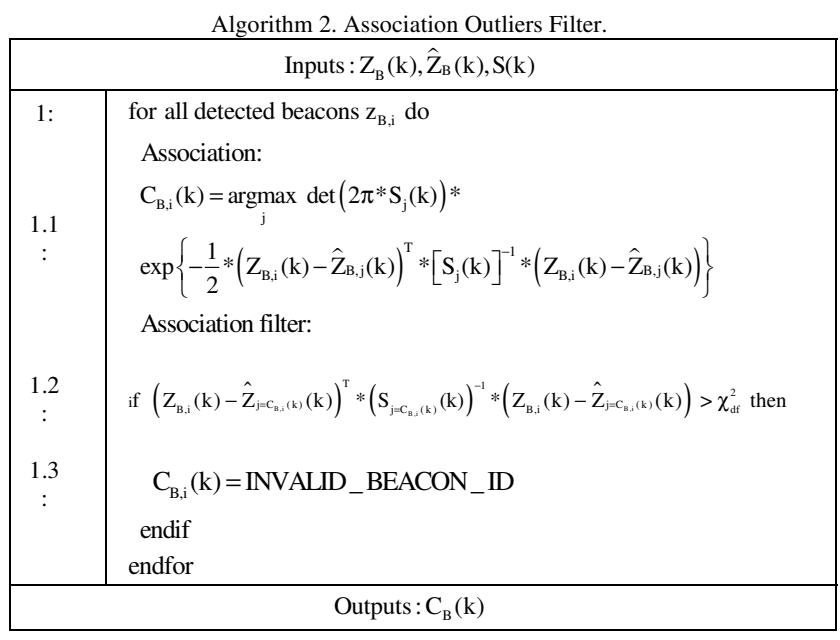

Step $1.1 \mathrm{Z}_{\mathrm{B}, \mathrm{i}}$ is related to $\mathrm{M}_{\mathrm{B}, \mathrm{i}}$ that maximizes the probability. Each $j$ of $\mathrm{M}_{\mathrm{B}}$ map calculates the probability distribution function $\mathrm{Z}_{\mathrm{B}, \mathrm{i}}$ (likelihood). The $i$ observation is related to $j$ when maximized (maximum likelihood). For more details see [9].
Step 1.2: $\chi_{\mathrm{df}}^{2}$ is a constant that depends of $P_{G}$ minimum threshold validation (Fig. 3).This value is related to the inverse function of the chi square quadratic form probability for two degrees of freedom. An enhanced approach was based on [9] in which is proposed a likelihood filter. Further Fig. 12 shows the probability of reflector detection of $95 \%$.

As conclusion, the Kalman Filter was used to implement the sensor fusion task between detected reflectors and odometry data. The robustness of this outliers filter algorithm can be emphasized due to the combination of detector and association filters.

\section{PRACTICAL RESULTS}

First, the time requirements are validated: $5 \mathrm{~ms}$ to process 760 beams of laser with an Intel T4300 processor with $1.2 \mathrm{GHz}$ clock speed. This is a compatible time to perform decision tasks and control. Robot position repeatability on target position was about $10 \mathrm{~mm}$.

The assembled industrial mobile robot was used to perform all measurements and tests. The tests were conducted in the laboratories of the Faculty of Engineering of University of Porto and in the ADIRA company as present in next subsections. As it can be seen, the developed algorithm allows rejecting outliers objects that could interfere in localization.

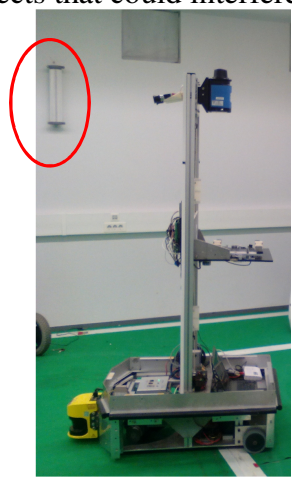

Fig. 7. Developed Mobile platform to perform security laser and triangulation ground truth comparison (reflector highlighted in red circle).

\section{A. Precision Results}

As a way to validate our approach with the ground truth it was used another mobile robot (see Fig. 7) which was equipped with a commercial navigation laser based on triangulation (SICK NAV350). This laser does not only provides the robot pose but also the reflectors relative position $\left(\mathrm{Z}_{\mathrm{B}}\right.$ of Fig. 3$)$.

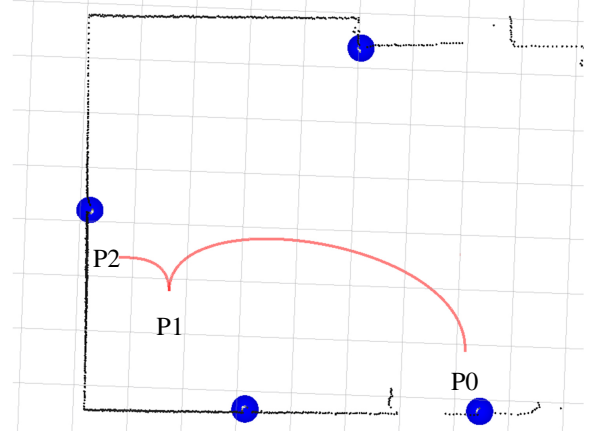

Fig. 8. Map of the AGV path testing. Grid size one meter. 
In Fig. 8 we present four reflectors (blue circles) installed in the walls and the path followed by the mobile robot (red line). It starts moving forward from $\mathrm{P} 0$ reaching $\mathrm{P} 1$ and then moving backward to P2. This movement allow to compare the presented method (based on a security laser with the ground truth). Reflectors in this experience are not affected by outliers.

TABLE I. PRECISION ANALYSIS

\begin{tabular}{|c|c|c|c|c|c|c|}
\hline & \multicolumn{3}{|c|}{ Position error $(\mathrm{m})$} & \multicolumn{3}{c|}{ Orientation error $\left(^{\circ}\right)$} \\
\hline F.O.V.( $\left(^{\circ}\right)$ & Mean & Std. Dev. & Max. & Mean & Std Dev. & Max. \\
\hline 360 & 0.0016 & 0.0023 & 0.016 & -0.0034 & 0.020 & 0.087 \\
\hline 180 & 0.0026 & 0.0039 & 0.035 & -0.0035 & 0.063 & 0.46 \\
\hline
\end{tabular}

As expected, the low positioning errors (average and standard deviation) was observed as presented in TABLE I. It also shows that the proposed algorithm stays robust in terms of precision when the FOV is reduced to $180^{\circ}$ (measures inside [$\left.90^{\circ},+90^{\circ}\right]$ are considered) as it happens in security lasers scanners.

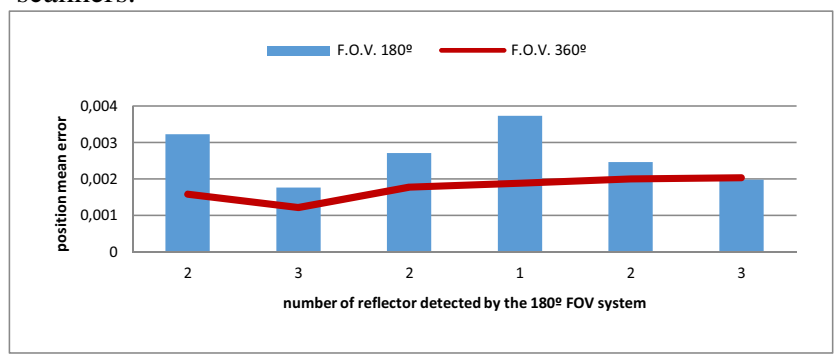

Fig. 9. Comparison of error between the $360^{\circ}$ FOV triangulation laser (red line) and $180^{\circ} \mathrm{FOV}$ (security laser).

Blue bars of Fig. 9 present the average error whereas the ground truth error is shown by red line. Initially, the left bar is related to the start of movement (P0) while the right blue bar corresponds to the last movement of the mobile robot (P2).

As it can be seen, precision of the proposed system is not considerably affected by the reduction of the FOV. This results allow to conclude that the use of Kalman Filter is an advantage and that the triangulation system should be used only in situations that allows three or more visible reflectors (five recommended). Our system (odometry and laser data fusion) is capable of positioning the robot even with three or less reflectors. The commercial navigation laser with $360^{\circ} \mathrm{FOV}$ always sees four reflectors.

\section{B. Robustness analisys-Outliers rejection}

This subsection presents the mobile robot equipped with the security laser of Fig. 1 navigating in a real industry environment with several outliers and adversities, like Fig. 10.

In the scope of Project PRODUTECH PSI PPS3 it was implicit a public demonstration of the developed system which was used to validate our developed localization system in an industrial environment. Fig. 10 shows the demonstration scenario. The autonomous robot localization tests were developed during 6 hours. The robot mission was to transport tables between workstations with a required positioning precision of $1 \mathrm{~cm}$ and 3 degrees and a speed of $0.5 \mathrm{~m} / \mathrm{s}$. This demanding mission had an increased difficulty since lots of people were circulating in the path of the robot and the irregular floor decreased (to $4 \mathrm{~m}$ ) the laser visibility. This environment increased the outliers' number as show in Fig. 11 where dark blue circles are the reflectors acquired during the movement from $\mathrm{P} 0$ to $\mathrm{P} 2$ (backward from $\mathrm{P} 0$ to $\mathrm{P} 1$ and forward from P1 to P2). Security laser data is represented by the yellow and red points: the yellow ones are the reflectors (inliers) and red ones are the outliers rejected by the detector and filter algorithm. Note that it is a compiled information and not all reflectors are seen simultaneously.

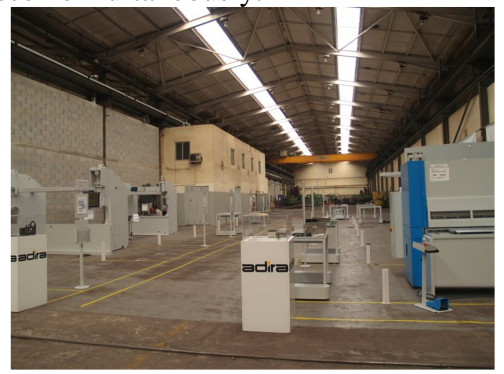

Fig. 10. Industrial environment scenario where localization tests were developed.

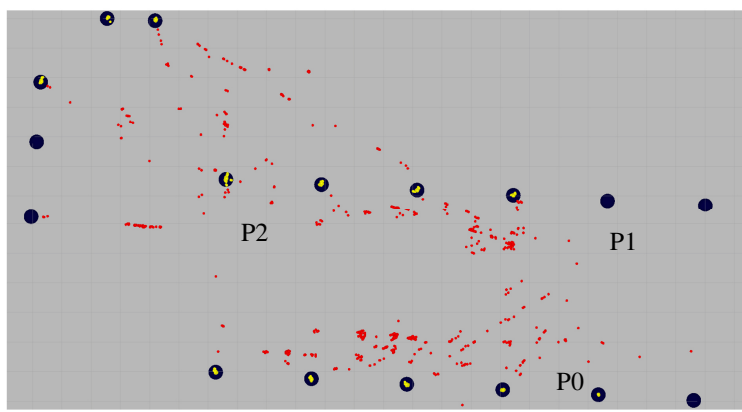

Fig. 11. Map of the mobile robot path: red points are outliers, yellow points are the reflectors detection and dark blue circles are the reflectors position. Grid size one meter.

Figure 12 presents a screenshot of the developed interface application during the tests. There can be seen: reflectors map $\left(\mathrm{M}_{\mathrm{B}}\right.$ represented in dark blue circles), co-variance of the estimated robot position (red ellipse in the right upper corner) and reflectors zones for the accepted measures (more than $95 \%$ probability)with blue ellipses. Red dots (the left bottom corner) show objects measured as reflectors but are rejected by filter once they are outside of $95 \%$ of accepted threshold: there is a certain area around the reflectors that supply the outliers filter. Outside reflectors are ignored by the outliers filter. Black points are the measured distances by the security laser $\left(Z_{L}\right)$.

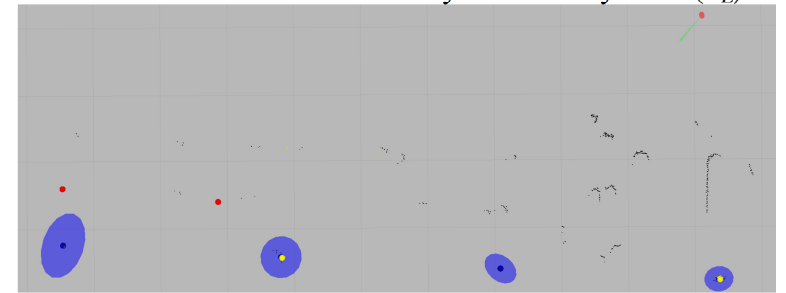

Fig. 12. - Screenshot of the real results during localization tests it is possible to notice a large number of positive false measures with a security laser. The developed robust outliers filter algorithm becomes the most important task in this approach. It is applied in two ways: detector filter and association filter. 
In order to highlight and compare the developed filtering system, Fig. 13. and Fig. 14. presents the localization system for positioning and orientation respectively without any filter (blue line), only with detection filter (green line) and finally with both (detector and association filter) in red line. As it is easy to understand, the absence of filtering system produces noisy and unstable results. The detector filter improves the result of positioning but it still lacks stability and introduces some error. Finally, the use of both filters allows to perform a stable and accurate measure without error and changes.

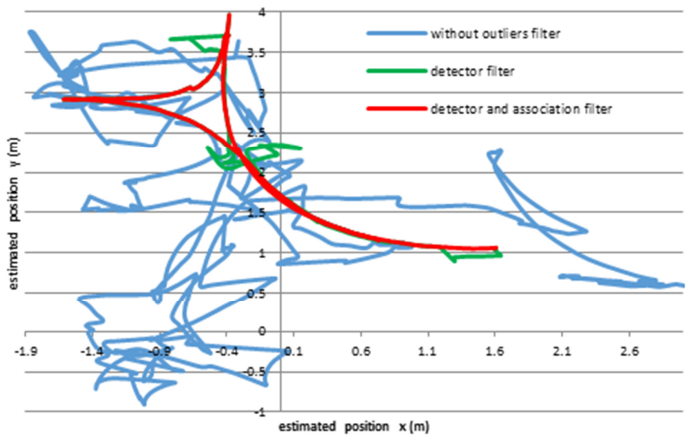

Fig. 13. Comparison of filtering rejection outliers. Positioning.

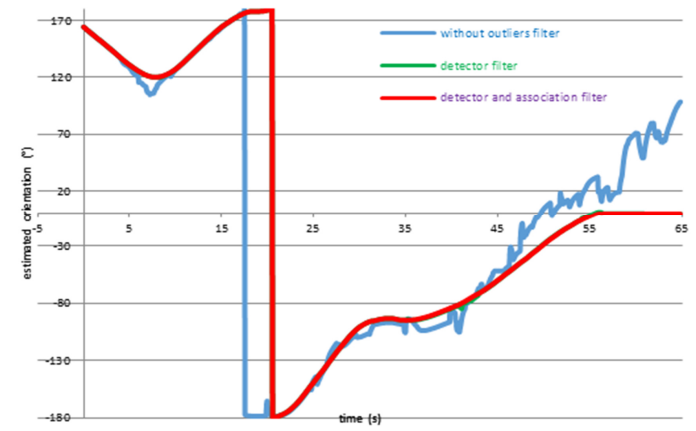

Fig. 14. Comparison of filtering rejection outliers. Orientation.

Videos containing the localization system interface during the tests can be downloaded at these links:

- Without outliers filter: http://youtu.be/wCv9qVTSICg

- Detector filter: http://youtu.be/iTCb5UR6CRE

- Detector and association filter: http://youtu.be/4_Io52ORvOE

Videos during the tests where the mobile robot is in transporting mission can be downloaded at these links:

- Laboratory: http://youtu.be/6SQ31lbTSFk

- Adira industrial environment: http://youtu.be/KI3rkx7vS2Y

\section{CONCLUSION AND FUTURE WORK}

The developed localization system based on a security laser scanner was applied in an industrial AGV. The experiments allow to confirm that in real industrial environment there are advantages in the proposed localization algorithm over the current laser positioning systems.

The Extended Kalman Filter was applied as a multi fusion sensor system in order to combine the odometry information and the result of the developed system. As final remark the presented algorithm ensures precision $(10 \mathrm{~mm}$ of best depending on the number of reflectors) and fast computation time $(5 \mathrm{~ms})$.

As a future direction, enhancing the beacon detection system (increasing the number of detected features) will allow to benefit of better robustness and precision. Moreover, implementing redundant methods will improve the good results of the presented system (minimizing the ambiguity in symmetric layouts and deal with the kidnapping problem).

\section{ACKNOWLEDGMENT}

The work presented in this paper, being part of the Project "NORTE-07-0124-FEDER-000060" is financed by the North Portugal Regional Operational Programme (ON.2 - O Novo Norte), under the National Strategic Reference Framework (NSRF), through the European Regional Development Fund (ERDF), and by national funds, through the Portuguese funding agency, Fundação para a Ciência e a Tecnologia (FCT).

\section{REFERENCES}

[1] O. Wulf, D. Lecking and B. Wagner, Robust Self-Localization in Industrial Environments based on 3D Ceiling Structures, Proceedings of the 2006 IEEE/RSJ International Conference on Intelligent Robots and Systems, 2006

[2] J. Liu, B. Yin and X. Liao, Robot Self-localization with Optimized Error Minimizing for Soccer Contest, Journal Of Computers, Vol. 6, no. 7 , 2011.

[3] M. Quigley, B. Gerkey, K. Conley, J. Faust, T. Foote, J. Leibs, E. Berger, R. Wheeler, and A. Y. Ng, "ROS: an open-source Robot Operating System,"In Proc. Open-Source Software workshop of the International Conference on Robotics and Automation, Kobe, Japan, May, 2009.

[4] M. Lauer, S. Lange and M. Riedmiller, Calculating the Perfect Match An Efficient and Accurate Approach for Robot Self-localization, RoboCup Symposium, pp. 142-53, Osaka, Japan, 13-19 July, 2005.

[5] S. Thrun, W. Burgard and D. Fox, Probabilistic Robotics, Massachusetts Institute of Technology, 2006.

[6] G. Grisetti , C. Stachniss and W. Burgard, "Improved Techniques for Grid Mapping with Rao-Blackwellized Particle Filters", IEEE Transactions on Robotics, Vol. 23, No. 1, pp. 34-46, February 2007.

[7] J. Borenstain, H. R. Everett, L. Feng and D. Wehe, "Mobile Robot Positioning and Sensors and Techniques", Journal of Robotic Systems, Special Issue on Mobile Robots, Vol. 14 No. 4, pp. 231 249, 1996.

[8] Ronzoni, D., Olmi, R., Secchi, C., \& Fantuzzi, C., AGV global localization using indistinguishable artificial landmarks, in IEEE International Conference on Robotics and Automation (pp. 287-292). Shanghai: IEEE. doi:10.1109/ICRA.2011.5979759, 2011.

[9] Sebastian Thrun, Wolfram Burgard, Probabilistic Robotics (Intelligent Robotics and Autonomous Agents series), 2005.

[10] Eliazar, A.I. and Parr, R. Learning probabilistic motion models for mobile robots, Proceedings of International Conference on Machine Learning, 2004.

[11] Héber Sobreira, Miguel Pinto, António Paulo Moreira, Paulo Gomes Costa, José Lima, Robust Robot Localization Based on the Perfect Match Algorithm, Proceedings of the 11th Portuguese Conference on Automatic Control Lecture Notes in Electrical Engineering Volume 321, 2015, pp 607-616, Portugal, 2014. 